\title{
Effect of Endoscopic Argon Plasma Coagulation on Gastrointestinal Blood Loss Due to Portal Hypertensive Gastropathy
}

\author{
Alaa-Eldeen Hashim¹, Samy Zaky¹, Mahmoud Saad Berengy²*, Tarek Emran ${ }^{3}$ and Mohamed \\ Hegazy $^{1}$
}

${ }^{1}$ Department of Tropical Medicine, Damietta Faculty of Medicine, Al-Azhar University, Egypt

${ }^{2}$ Department of Internal Medicine, Damietta Faculty of Medicine, Al-Azhar University, Egypt

${ }^{3}$ Department of Clinical Pathology, Damietta Faculty of Medicine, Al-Azhar University, Egypt

*Corresponding author: Mahmoud Saad Berengy, Department of Internal Medicine, Damietta Faculty of Medicine, Al-Azhar University, Egypt, Tel: +20 102722 7142; E-mail: mahmoudberengy78@yahoo.com

Rec date: Mar 13, 2017; Acc date: Mar 30, 2017; Pub date: Apr 1, 2017

Citation: Hashim AE, Zaky S, Berengy MS, Effect of Endoscopic Argon Plasma Coagulation on Gastrointestinal Blood Loss Due to Portal Hypertensive Gastropathy. J Clin Gastroenterol Hepatol 2017, 1: 2.

\section{Abstract}

Background: Portal hypertensive gastropathy (PHG) is one of the clinical conditions that can cause chronic gastrointestinal hemorrhage in patients with cirrhosis, manifested by chronic anemia. It is generally diagnosed on the basis of endoscopic features. A new thermal modality has recently been introduced to endoscopy: the argon plasma coagulator (APC). Hemostasis is a main target for the use of APC and its role in ceasing PHG related acute and chronic bleeding is being efficient and safe when studied in the past few years.

Aim of the study: To evaluate the role of argon plasma coagulation in comparison with non-selective beta blockers for the treatment of chronic blood loss and iron deficiency anemia in cirrhotic patients with severe portal hypertensive gastropathy.

Patients and methods: This study included 112 cirrhotic anemic patients who had severe PHG. The patients were divided into two groups, group (A) included 56 patients who had argon plasma coagulation (APC) sessions and group (B) included 56 patients who received propranolol as oral therapy. Response to the treatment was assessed by the change in hemoglobin and iron parameters over the following three months after the start of therapy.

Results: On comparing laboratory investigations between 1st visit and one, two and three months after the 1st visit in group (A) patients, there was a highly significant increase in hemoglobin level, serum iron and serum ferritin with a significant decrease in TIBC. This means that there was an overall improvement of anemia and iron deficiency. On comparing laboratory investigations between 1st visit and one, two and three months after the 1st visit in the use of beta-blockers (group B) is associated with the gradual increase in the mean of hemoglobin, serum iron, and serum ferritin and gradual decrease of TIBC. There was statistically significant difference between the two groups as regards the changes in hemoglobin and iron study after one, two and three months respectively when compared with the first visit that means that the increase in hemoglobin, serum ferritin, serum iron is more in APC group patients than beta-blockers group patients and the decrease in TIBC is more in APC group patients than beta-blockers group patients, so the improvement in hemoglobin and iron study variables is better in APC group patients than detected in beta-blockers group patients. Taking into account portal vein dilatation and decreased flow velocity, two physiological changes associated with portal hypertension, the "congestion index" was used to assess portal hypertension which is the ratio of portal vein crosssectional area $\left(\mathrm{cm}^{2}\right)$ to mean portal vein flow velocity $(\mathrm{cm} / \mathrm{sec})$. In our study the mean congestion index of the main portal vein was $0.18(\mathrm{~cm} \times \mathrm{sec})$ and it is significantly higher in bleeder than in non-bleeder group and at a cutoff limit of congestion index of portal vein $0.24(\mathrm{~cm} \times$ sec) the sensitivity is $83 \%$ and specificity is $96 \%$ in discriminating bleeder from non-bleeder group.

Conclusion: The use of either argon plasma coagulation (APC) or beta-blockers in controlling the chronic blood loss from congestive gastropathy shows significant improvement in hemoglobin and iron study variables with both but the significant improvement in APC group patients than in beta-blockers group patients recommended the use of $A P C$ to control the chronic blood loss from congestive gastropathy.

Keywords: Argon plasma coagulation; Portal hypertensive gastropathy; Blood loss

\section{Introduction}

Portal hypertensive gastropathy (PHG) is characterized by typical gastric mucosal lesions presenting in patients with portal hypertension. The typical location is in the gastric fundus and upper body of the stomach although it can affect 
the whole stomach and even other areas of the gastrointestinal tract, such as the small bowel or the colon [1]. PHG is one of the major health problems as its prevalence in patients with portal hypertension has been reported to vary between $20 \%$ and $80 \%$. The wide variation in the reported prevalence is most likely due to differences in the study population specifically, the proportion of patients with noncirrhotic portal hypertension, the severity of the underlying liver disease, and the proportion of patients with previous endoscopic treatment. A higher rate of PHG is observed in patients with more severe liver disease and in patients who have had previous endoscopic treatment with sclerotherapy or endoscopic variceal ligation [2]. The diagnosis of PHG, and by extension portal hypertensive enteropathy (similar mucosal changes at other sites of the gastrointestinal tract) is established when the characteristic endoscopic findings are observed in patients with portal hypertension. PHG is classified as mild when only the snake-skin mosaic pattern is present or severe when, in addition to the mosaic pattern, flat or bulging red marks or black-brown spots are observed [3].

The clinical relevance of this classification has been established since patients with severe PHG are more likely to have acute bleeding or chronic anemia than patients with mild PHG [4]. Furthermore, this classification is reproducible as a fairly good concordance between observers has been shown, especially regarding the mosaic pattern and red marks [5].

Studies that evaluate the probability of bleeding from PHG report an incidence between $2.5-30 \%$, with the greatest incidence observed in patients with severe PHG. Diagnosis of acute hemorrhage from PHG is established when active bleeding from gastropathy lesions or non-removable clots overlying these lesions is observed or when there is PHG and no other cause of acute bleeding can be demonstrated after thorough evaluation of the gastrointestinal tract [4].

Treatment of PHG relies on two main pillars. Firstly, general measures used in gastrointestinal bleeding independent of cause should be applied and secondly a specific approach to treat the cause of the gastrointestinal bleed should be undertaken. The most effective specific treatments in patients with PHG are those aimed at reducing portal pressure. In the most frequent context of chronic blood loss, iron supplementation should be provided in order to counteract the continuous depletion of iron deposits. The most effective specific therapy is that aimed at reducing portal pressure. Nonselective beta-blockers have been shown to decrease bleeding from both acute and chronic forms of hemorrhage from PHG. Propranolol is given at an initial dose of $20 \mathrm{mg}$ BID, gradually escalated to $160 \mathrm{mg}$ BID or the maximum tolerated dose according to heart rate (50-55 bpm) or secondary effects (lightheadedness, asthenia, etc). This therapy should be maintained as long as the patient continues to have portal hypertension. Use of other pharmacologic agents in PHG such as other medications that primarily treat bleeding, including antifibrinolytic medications such as tranexamic acid [6].

Since portal hypertension is the main underlying factor that leads to the development of PHG, shunt therapies have been evaluated as salvage therapies. Data has been reported regarding the use of both the transjugular intrahepatic portosystemic shunt (TIPS) and shunt surgery. Due to the morbidity associated with shunt surgery, particularly in patients with cirrhosis, its role may be more relevant in patients with non-cirrhotic portal hypertension [7].

Argon plasma coagulation and electrocautery have both been used to stop bleeding from ectatic vessels, and to attempt to obliterate the vessels, but have limited utility if the disease is diffuse [8].

Zakareya et al. found favorable results on chronic blood loss using Argon plasma coagulation (APC) [9]. The literature reports suggest both regression of portal hypertensive gastropathy on endoscopic images and improvement in bleeding after TIPS [10]. Finally, cryotherapy involves the use of pressurized carbon dioxide administered through the endoscope to freeze and destroy tissue in a focal area [11].

Due to many side effects and poor compliance of patients to beta blockers and morbidity associated with shunt surgery, Herrera et al. evaluated the use of endoscopic treatment of PHG with argon plasma coagulation (APC) and recommended that this endoscopic approach could be considered in patients in whom severe recurrent bleeding occurs despite betablockers and who are not candidates for TIPS [12].

\section{Aim of the Work}

The aim of the study is to evaluate the use of argon plasma coagulation for the control of chronic blood loss and iron deficiency in patients with portal hypertensive gastropathy.

\section{Patients and Methods}

This prospective study was carried out on 112 cirrhotic patients (67 males and 45 post-menopausal females). All of them had portal hypertensive gastropathy (PHG) and anemia. According to the type of treatment of the gastric blood loss and its consequent anemia, we divided the 112 patients into 2 equal groups: Group A included fifty-six patients treated with Argon Plasma Coagulation (APC); and Group B included fifty-six patients treated with beta blockers (propranolol 20-60mg twice daily with average dose $40 \mathrm{mg}$ twice daily according to the response: heart rate $(50-55 \mathrm{bpm})$ or secondary effects (light-headedness, asthenia, etc).

\section{Inclusion criteria included the following}

1) Chronic liver disease diagnosed clinically and confirmed by liver function tests and abdominal ultrasonography, 2) Severe PHG diagnosed by upper gastrointestinal endoscopy according to De Franchis's classification of PHG (2000) "mosaic pattern superimposed by red signs or any other red signs" and 3) Anemia with Hemoglobin level less than $10 \mathrm{gm} / \mathrm{dl}$.

\section{Exclusion criteria included the following}

1) Menstruating females; and patients who: a) were less than 18 or more than 60 years old, b) had bled from esophageal and/or gastric varices in the last 6 months, c) had 
risky varices, d) had taken NSAIDs in the last month, e) have esophgitis, gastritis and/or duodenitis, F) have esophageal, gastric, duodenal erosions and/or ulcerations, g) have esophageal, gastric and duodenal mass or tumor, h) have hiatus hernia, i) have ankylostoma infestation, j) have recurrent epistaxis, k) have hematuria, L) had received iron therapy or blood transfusion in the last 3 months, $\mathrm{m}$ ) have internal and/or external piles and/or anal fissure, n) patients with severe disturbance of the coagulation profile, o) uremic patients and $\mathrm{p}$ ) with malnutrition.

Patients included in this study were selected from both Tropical and Internal Medicine Departments at Al-Azhar University Hospital in New Damietta city. After diagnosis of all selected patients as having severe PHG, other different possible causes of anemia were excluded clinically and by laboratory investigations.

The study protocol conforms to the ethical guidelines of the 1975 Declaration of the Helsinki.

The selected groups of patients were subjected to the following: 1) Full history taking, 2) Complete clinical examination and 3) Laboratory investigations: a) Liver function tests (alanine transaminase (ALT), aspartate transaminase (AST), bilirubin (total and direct) and albumin, b) Complete blood picture, c) Prothrombin time-INR, d) Iron profile (serum iron,TIBC and ferritin).

\section{Laboratory Tests}

$5 \mathrm{ml}$ of venous blood were taken from all patients. Blood samples were divided into EDTA, citrate and plain tubes. Liver functions, serum iron and TIBC were measured on an automated biochemistry analyzer (Beckman Coulter AU480). Complete blood picture was done using Sysmex automated hematology analyzer (Sysmex Corporation, Japan). Ferritin level was measured by chemiluminescence, with Immulite (Diagnostic Products Corporation; Los Angeles, CA, USA).

Child's Pugh scoring: was applied for cirrhotic patients to obtain the severity of liver cell affection, as described by Pugh et al. [13]. In addition, color Doppler ultrasound on main portal vein to calculate congestion index as follows: "The Doppler probe was at a fixed angle of $55^{\circ}$. In the Doppler-mode, ultrasound waves were emitted and received by a single probe at a frequency of $2.27 \mathrm{MHz}$ and with a repetition frequency of $4 \mathrm{kHz}$ or $6 \mathrm{kHz}$. Spectral analysis was performed using fast Fourier transform, and the Doppler shift frequency signal was recorded on paper using a line scan recorder. All patients were studied during fasting and at rest in a semi-reclining posture of about $30^{\circ}$. To decrease the effect of respiration on the portal blood flow, all measurements were obtained during quiet respiration, avoiding deep respiration or breath-holding. Doppler data were obtained while scanning the portal vein along its longitudinal axis and with the sample volume set on the middle of the portal vein trunk. Just after the Doppler signals were recorded, the portal vein cross sectional area was measured from the B-mode image of the portal vein while scanning perpendicular to the long axis of the portal vein. The cross-sectional area was calculated from the formula for the cross-section of an ellipse. The angle between the Dopplermode ultrasound beam and the blood vessel was then measured on the B-mode image. The $\mathrm{Cl}$ was calculated using the following equation: $\mathrm{Cl}=$ (Cross-sectional area of portal vein)/ (Blood flow velocity of portal vein) [14].

Finally, upper gastrointestinal endoscopy was done as the following: Sedation for endoscopy was done using intravenous midazolam in a dose of 2.5-7.5 mg. Upper gastrointestinal endoscopy was done by a single endoscopist. Stressing on the degree of congestive gastropathy according to de Franchis classification, grade of esophageal varices, fundal extension, stomach and duodenum for gastritis or duodenitis respectively, erosions and ulcerations. Accordingly, congestive gastropathy was classified as follows: Mild: when mosaic like pattern (MLP) of mild degree (without redness of areola) is present; Severe: when the mosaic like pattern (MLP) is superimposed by red signs or if any other red sign is present [15].

Moreover, argon Plasma Coagulation: Done to group A. The APC equipment consisted of APC probe advanced from the end of the working channel of the endoscope, a gas source and a high-frequency generator. The argon gas flow was set at a rate of $2.5 \mathrm{~L} / \mathrm{min}$. The electrical power output was adjusted at "60 $W^{\prime \prime}$ which was safe in relation to the local risk of perforation at the site of application of the APC to the gastric mucosa. Electrical spark could be easily elicited by pressing the blue foot pad connected to APC equipment. APC was applied to all areas of visible red spots of congested gastric mucosa with approximately 3-5 $\mathrm{mm}$ distance between APC probe and gastric mucosal lesion for about 1-2 seconds. The end point of successful endoscopic therapy was production of a white coagulum. APC was done in a single session in patients who have localized PHG and in multiple sessions in patients who have diffuse PHG where endoscopy was repeated after 2 weeks, and further sessions were performed as needed.

During period of follow up beta blockers, hematenics, multivitamins and blood transfusion were not allowed.

\section{Statistical Analysis}

Data were analyzed by statistical package for social science (version 16.0) (SPSS Inc., Chicago, Illinois). Quantitative data were expressed as mean \pm standard deviation (mean \pm SD). General linear model was used to test differences at different times using repeated measures analysis of variance (ANOVA). Paired t-test was used to test before and after paired observations. Qualitative data were expressed as numbers and percentages and analyzed by chi-square $\left[\mathrm{x}^{2}\right]$ test. For calculated of cut -off values, the Receiver Operator Curve (ROC) was calculated and the best cut off was determined. For correlation between two paramours, the Pearson's correlation coefficient ( $r$ ) was calculated; it was inverse if the sign is negative and proportional if the sign is positive. Level of significance was set to P-value $<0.05$. 


\section{Results}

This Per Protocol (PP) and Intension to Treat (ITT) prospective study was carried out on 112 cirrhotic patients, 6 patients of them were escaped during the first month of follow up and 106 patients were continued regularly in the study. All of them had portal hypertensive gastropathy (PHG) and anemia due to chronic blood loss as evidenced by occult blood in stool. All patients were included in the study after signing an informed consent. Mean age in group I was 47.6 years and 46.3 years in group II, and there was no statistical significant difference between groups. The study conducted on 67 (59.8\%) males and 45 (40.2\%) females and $92.1 \%$ of patients were from rural areas and $17.9 \%$ of patients were from urban areas. The most common cause is HCV infection either alone $40.2 \%$ or associated with bilharziasis $50.9 \%$ and bilharziasis alone in only $8.9 \%$ of patients. History of previous endoscopic interventions (sclerotherapy and/or band ligation) showed statistically insignificant difference between the groups $(78.6 \%$ compared to $82.2 \%$ ). On the other hand, there was statistically insignificant difference between APC group and beta-blockers group as regards the ALT, AST, total and direct bilirubin, serum albumin, INR, hemoglobin, hematocrit, WBCs and platelets, but there is significant increase in serum iron, ferritin and significant decrease in TIBC in APC group when compared to beta blocker group. The ultrasound findings of portal hypertension (ascites, spleen and hilar collaterals) revealed that, there was statistically insignificant difference between the two groups. Ascites found in 97 patients (86.6\%), splenomegaly detected in 104 patients (92.9\%), splenectomy in 6 patients $(5.4 \%)$, and normal size spleen in only 2 patients $(1.7 \%)$ and hilar collaterals found in 16 patients (14.3\%) (Table 1).

In the present work, the mean of congestion index in both groups was $0.18(\mathrm{~cm} \times \mathrm{sec})$ statistically insignificant differences between the two groups regarding congestion index and blood flow volume but there is significant difference between the two groups regarding blood flow velocity in the portal vein. Results of the present study demonstrated, that there was 28 (25\%) patients with no esophageal varices 22 patients of them were eradicated previously and 6 patients had no history of esophageal varices detection, 27 (24.1\%) patients had grade I, $43(38.4 \%)$ patients had grade II and 14 (12.5\%) patients had grade III, and there was statistically insignificant difference between the two groups. Furthermore, there was no significant difference between both groups as regard to fundal varices. It was found in (18.7\%) in total number of patients. As regard the severity of portal hypertensive gastropathy according to NIEC, black brown spots accounts about (50.8\%) in both groups, cherry red spots in $(43.8 \%)$ and red point lesions in (5.4\%) in both groups and there was statistically insignificant difference between the two groups as shown in Table 2. The sites of portal hypertensive gastropathy showed statistically insignificant difference between the two groups, also it shows that fundus is affected in most of the patients 97 patients $(86.6 \%)$ either alone $(19.6 \%)$ or with other parts of the stomach) then antrum in 78 patients (69.6\%) either alone $(6.2 \%)$ or with other parts of the stomach) then the body 68 patients (60.7\%) always with other parts of the stomach). The body always affected with other parts of the stomach and we didn't detect it affected alone. In addition, hepatic functional reserve measured by ChildPugh's classification of all patients showed statistically insignificant difference between the two groups as regard Child class. By the Child Pugh classification, we divided the patients into: Child $A$ that included 15 patients (13.4\%), Child B included 41 patients (36.6\%) and Child C included 56 patients (50\%) (Table 2). In APC group, there was significant increase of hemoglobin, serum iron, serum ferritin and significant decrease of TIBC, at all postoperative check points when compared to pre-treatment values in both ITT and PP. similar findings were found in beta-blockers group. In addition, there was significant increase of hemoglobin, serum iron, serum ferritin and significant decrease of TIBC in group $A$ when compared to group $B$, after treatment, two and three months after treatment (Table 3).

In the present work, there was no significant difference between different esophageal grades, and different child grades, different sites of congestive gastropathy as regard to congestion index (data not tabulated). On the other hand, there was significant decrease of blood flow velocity in study group before treatment and significant increase at 3 months after treatment, when compared to group B. In addition, there was significant increase of blood flow velocity in group $A$ and significant decrease in group $B$ at 3 months after treatment when compared to corresponding values before treatment. Similar findings were found for blood flow volume. Furthermore, at 3 months after treatment, there was significant decrease of congestion index in study group (16.0 \pm $0.07)$ when compared to group $B(0.20 \pm 0.05)$. At the same time, there was significant decrease of $\mathrm{Cl}$ in group $\mathrm{A}$ and significant increase in group $B$ at 3 months when compared to their corresponding values before treatment. However, postoperative child grading had no significant difference in either groups when compared to values before treatment (data not tabulated).

As regard to re-bleeding in group A, it was $28.8 \%, 21.4 \%$ and $16.1 \%$ in group $A$ at one, two and three months after treatment; while it was $39.3 \%, 32.1 \%$ and $30.4 \%$ in group B respectively; with no significant difference between groups. By occult blood, the percentages of rebleeding was $30.0 \%, 24.0 \%$ and $18 \%$ in group $A$ after one, two and three months respectively, compared to $39.3 \%, 32.1 \%$ and $30.4 \%$ in group B respectively with no significant difference between both groups.

As regard complications in group A, gaseous distension was reported in $58.9 \%$ and pain at treatment site in $37.5 \%$. In group B, complications were asthenia in $60.7 \%$ and headache in $50.0 \%$.

At the end of the study the patients divided according to continuous blood loss in stool into two group's bleeder group which included 26 patients (32.2\%) and non-bleeder group which included 86 patients (76.8\%). There was no significant difference between bleeders and non-bleeders as regard all studied variables except significant increase of congestion index in bleeders before and after treatment $(0.26 \pm 0.02,0.26$ 
\pm 0.06 respectively) when compared to non-bleeders $(0.16 \pm$ $0.05,0.16 \pm 0.02$ respectively). However, no significant difference after three months of treatment when compared to values before treatment in each group. Receiver operating characteristics (ROC) curve estimation of congestion index in determination of re-bleeding show that at a cutoff limit 0.23 (cm $\times \mathrm{sec}$ ) the sensitivity is $95 \%$ and the specificity is $91 \%$, and at a cutoff $0.25(\mathrm{~cm} \times \mathrm{sec})$ the sensitivity is $70 \%$ and the specificity is $100 \%$ while the most suitable cutoff value is 0.24 (cm $\times \mathrm{sec}$ ) with sensitivity is $83 \%$ and the specificity is $96 \%$. In multivariate analysis, the most sensitive predictor of bleeding from portal hypertensive congestive gastropathy was congestion index ( $p$-value $=0.005)$ then the severity of congestive gastropathy ( $p$-value=0.04) (Table 4 and Figure 1).

Table 1 Comparison between group A and B as regard to demographics, laboratory investigations and ultrasound.

\begin{tabular}{|c|c|c|c|c|}
\hline Variables & & Group A $(n=56)$ & Group B $(n=56)$ & $P$ value \\
\hline Age & & $47.6 \pm 5.55$ & $46.26 \pm 4.64$ & 0.18 (NS) \\
\hline Gender (M/F) & & $35(62.5 \%) / 21(37.5 \%)$ & $32(57.2 \%) / 24(42.8 \%)$ & $0.68(\mathrm{NS})$ \\
\hline Residence (R/U) & & $45(80.4 \%) / 11$ (19.6\%) & $47(83.9 \%) / 9(16.1 \%)$ & 0.50 (NS) \\
\hline \multirow{3}{*}{ Cause of $\mathrm{PH}$} & Viral & $21(37.5 \%)$ & \multicolumn{2}{|l|}{$24(42.9 \%)$} \\
\hline & Bilharzial & $4(7.1 \%)$ & $6(10.7 \%)$ & $0.34(\mathrm{NS})$ \\
\hline & Mixed & $31(55.4 \%)$ & $26(46.4 \%)$ & \\
\hline Previous intervention & & $44(78.6 \%)$ & $46(82.2 \%)$ & $0.80(\mathrm{NS})$ \\
\hline ALT (IU/L) & & $42.06 \pm 12.42$ & $42.58 \pm 15.33$ & 0.85 (NS) \\
\hline AST (IU/L) & & $39.84 \pm 11.51$ & $39.96 \pm 14.61$ & 0.96 (NS) \\
\hline Bilirubin (total) (mg/dl) & & $3.29 \pm 1.33$ & $3.12 \pm 1.06$ & 0.48 (NS) \\
\hline Bilirubin (direct) $(\mathrm{mg} / \mathrm{dl})$ & & $1.49 \pm 0.72$ & $1.42 \pm 0.57$ & 0.61 (NS) \\
\hline Albumin & & $2.60 \pm 0.54$ & $2.59 \pm 0.49$ & 0.92 (NS) \\
\hline INR & & $2.04 \pm 0.38$ & $1.04 \pm 0.30$ & 0.93 (NS) \\
\hline Hemoglobin (g/dl) & & $7.82 \pm 0.64$ & $7.63 \pm 0.65$ & 0.15 (NS) \\
\hline Hematocrit (\%) & & $26.32 \pm 3.78$ & $25.51 \pm 2.40$ & 0.06 (NS) \\
\hline WBCs & & $4.71 \pm 3.66$ & $3.58 \pm 1.73$ & 0.26 (NS) \\
\hline Platelets & & $92.27 \pm 47.87$ & $83.8 \pm 29.11$ & $0.052(\mathrm{~S})$ \\
\hline Serum iron (micg/dl) & & $50.36 \pm 2.72$ & $48.40 \pm 2.74$ & $0.001^{*}$ \\
\hline Serum ferritin (ng/ml) & & $9.12 \pm 0.45$ & $8.76 \pm 0.57$ & $0.001^{*}$ \\
\hline $\operatorname{TIBC}($ micg/dl) & & $471.66 \pm 20.37$ & $485.38 \pm 19.04$ & $0.001^{*}$ \\
\hline \multirow{3}{*}{ Ascites } & No & $9(16.1 \%)$ & $6(10.7 \%)$ & \multirow{3}{*}{0.56 (NS) } \\
\hline & Mild & $19(33.9 \%)$ & $23(41.1 \%)$ & \\
\hline & Moderate & $28(50.0 \%)$ & $27(48.2 \%)$ & \\
\hline \multirow{3}{*}{ Spleen } & Splenomegaly & $50(89.2 \%)$ & $54(96.4 \%)$ & \multirow{3}{*}{0.65 (NS) } \\
\hline & Splenectomy & $4(7.2 \%)$ & $2(3.6 \%)$ & \\
\hline & Normal & $2(3.6 \%)$ & $0(0.0 \%)$ & \\
\hline
\end{tabular}

Table 2 Comparison between group A and B as regard to Doppler ultrasound, upper GIT endoscopy.

\begin{tabular}{|l|c|c|}
\hline Variables & Group A (n=56) & Group B (n=56) \\
\hline Congestion index & $0.18 \pm 0.05$ & $0.18 \pm 0.07$ \\
\hline Blood flow volume & $1078 \pm 124$ & $1025 \pm 176$ \\
\hline Blood flow velocity & $9.23 \pm 1.54$ & $10.15 \pm 2.32$ \\
\hline
\end{tabular}




\begin{tabular}{|c|c|c|c|c|}
\hline \multirow[t]{4}{*}{ OV grade } & Eradicated & $14(25 \%)$ & $14(25 \%)$ & \multirow[t]{4}{*}{0.86 (NS) } \\
\hline & 1 & $15(26.8 \%)$ & $12(21.4 \%)$ & \\
\hline & II & $21(37.5 \%)$ & $22(39.3 \%)$ & \\
\hline & III & $6(10.7 \%)$ & $8(14.3 \%)$ & \\
\hline \multicolumn{2}{|l|}{ Fundal varices } & $8(14.3 \%)$ & $13(23.2 \%)$ & 0.29 (NS) \\
\hline \multirow{3}{*}{$\begin{array}{l}\text { Severity } \\
\text { Of Congestive } \\
\text { Gastropathy }\end{array}$} & Red point lesions & $2(3.6 \%)$ & $4(7.1 \%)$ & \multirow[t]{3}{*}{0.59 (NS) } \\
\hline & Cherry red spot & $25(44.6 \%)$ & $24(42.9 \%)$ & \\
\hline & Black brown spots & $29(51.8 \%)$ & $28(50 \%)$ & \\
\hline \multirow{6}{*}{$\begin{array}{l}\text { Site of } \\
\text { PHG }\end{array}$} & Fundal & $12(21.4 \%)$ & $10(17.9 \%)$ & \multirow[t]{6}{*}{0.84 (NS) } \\
\hline & Diffuse & $23(41.1 \%)$ & $25(44.6 \%)$ & \\
\hline & Antral & $2(3.6 \%)$ & $5(8.9 \%)$ & \\
\hline & Fundal and Antral & $8(14.3 \%)$ & $7(12.5 \%)$ & \\
\hline & Antral and body & $4(7.1 \%)$ & $4(7.1 \%)$ & \\
\hline & Fundal and body & $7(12.5 \%)$ & $5(8.9 \%)$ & \\
\hline \multirow{3}{*}{$\begin{array}{l}\text { Child } \\
\text { Classification }\end{array}$} & Child A & $9(16.1 \%)$ & $6(10.7 \%)$ & \multirow[t]{3}{*}{$0.63(\mathrm{NS})$} \\
\hline & Child B & $19(33.9 \%)$ & $22(39.3 \%)$ & \\
\hline & Child C & $28(50 \%)$ & $28(50 \%)$ & \\
\hline
\end{tabular}

Table 3 Comparison between groups A and B as regard to hemoglobin, serum iron, serum ferritin and TIBC after treatment, two and three months after treatment.

\begin{tabular}{|c|c|c|c|c|}
\hline Parameters & Variables & Group A $(n=56)$ & Group B $(n=56)$ & $P$ value \\
\hline \multirow{4}{*}{$\begin{array}{l}\text { After } \\
\text { Treatment }\end{array}$} & Hemoglobin & $8.50 \pm 0.68$ & $7.93 \pm 0.63$ & $<0.001^{*}$ \\
\hline & Serum iron & $63.64 \pm 3.33$ & $57.20 \pm 2.89$ & $<0.001^{*}$ \\
\hline & Serum ferritin & $10.13 \pm 0.44$ & $9.13 \pm 0.56$ & $<0.001^{*}$ \\
\hline & TIBC & $454.12 \pm 20.88$ & $477.28 \pm 18.88$ & $<0.001^{*}$ \\
\hline \multirow{4}{*}{$\begin{array}{l}\text { Two months } \\
\text { After treatment }\end{array}$} & Hemoglobin & $9.16 \pm 0.76$ & $8.25 \pm 0.62$ & $<0.001^{*}$ \\
\hline & Serum iron & $77.74 \pm 3.77$ & $68.84 \pm 4.03$ & $<0.001^{*}$ \\
\hline & Serum ferritin & $11.19 \pm 0.54$ & $9.41 \pm 0.59$ & $<0.001^{*}$ \\
\hline & TIBC & $410.18 \pm 26.92$ & $461.24 \pm 18.57$ & $<0.001^{*}$ \\
\hline \multirow{4}{*}{$\begin{array}{l}\text { Three months } \\
\text { After treatment }\end{array}$} & Hemoglobin & $9.92 \pm 0.74$ & $8.55 \pm 0.61$ & $<0.001^{*}$ \\
\hline & Serum iron & $84.90 \pm 3.88$ & $73.86 \pm 4.01$ & $<0.001^{*}$ \\
\hline & Serum ferritin & $11.80 \pm 0.53$ & $9.71 \pm 0.59$ & $<0.001^{*}$ \\
\hline & TIBC & $386.89 \pm 27.23$ & $445.12 \pm 18.88$ & $<0.001^{*}$ \\
\hline
\end{tabular}

Table 4 Multivariate analysis of predictors of bleeding from congestive gastropathy.

\begin{tabular}{|l|l|l|}
\hline Variable & O.R. & $\mathbf{9 5 \%} \mathbf{C l}$ \\
\hline Severity of Congestive gastropathy & 2.54 & $(1.02-6.30)$ \\
\hline Congestion index & 0.21 & $(0.16-0.28)$ \\
\hline
\end{tabular}




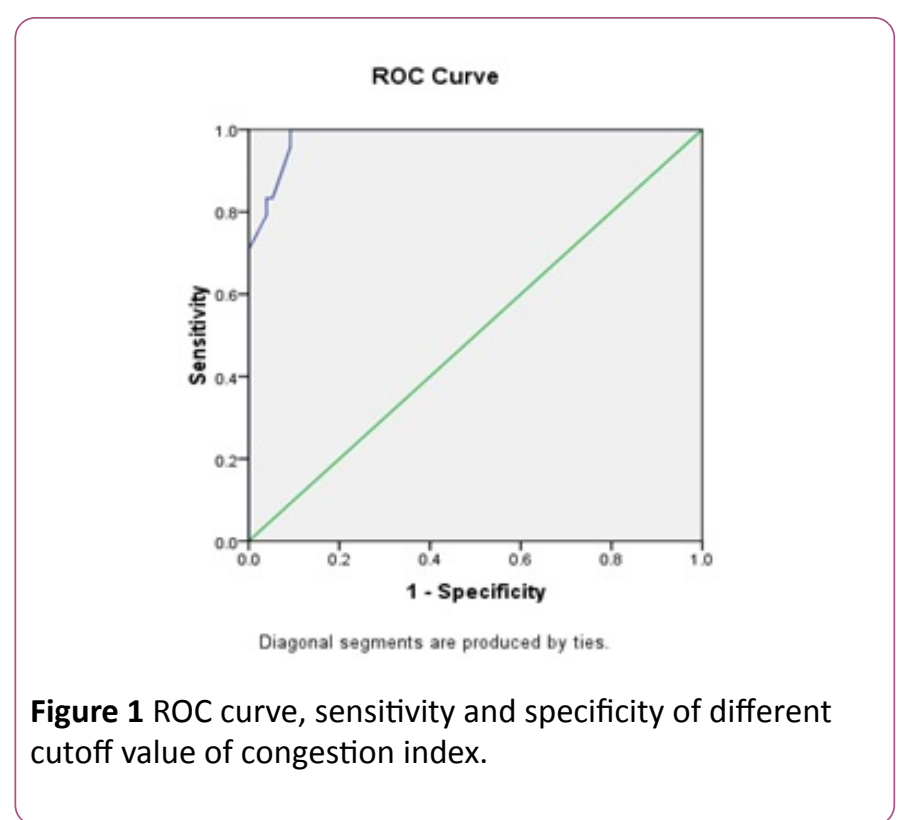

\section{Discussion}

This study was performed to evaluate the role of argon plasma coagulation in comparison with non-selective beta blockers for the treatment of chronic blood loss and iron deficiency anemia in cirrhotic patients with severe portal hypertensive gastropathy (PHG).

In present work, most common cause of liver disease was $\mathrm{HCV}$ infection either alone or associated with bilharziasis. The Egyptian Demographic Health Survey (EDHS), a cross sectional survey including hepatitis C virus (HCV) biomarkers, was conducted in 2008 on a large nationally representative sample. It estimated HCV prevalence among the 15-59 years age group to be $14.7 \%$ [16]. Accordingly, Egypt has the highest HCV prevalence in the world [17]. This unparalleled level of exposure to this infection appears to reflect a national level epidemic.

Results of the present study revealed that, the number of patients with Child score B and C (87\%) was found higher than the number of patients with Child score A (13\%). Bang et al. and Fontana et al. reported that PHG is more frequently observed in patients with more severe liver disease. The rate of endoscopic detection of PHG was found higher in the fundus and body than the antrum $[4,18]$. These results were found to be close to those reported by Bang et al. who stated that PHG lesions are most often typically found in the fundus and body (proximal to antrum) [4].

There is a positive history of variceal intervention (sclerotherapy or band ligation) found in $(80 \%)$ of patients including 24 patients ( $24 \%$ of total number of patients) were with variceal obliteration. Elwakil et al. suggested that variceal obliteration aggravates PHG [2]. This aggravation is probably caused by enhancement of gastric mucosal congestion by acute blockage of gastric mucosal blood flow. Endoscopic therapy has been associated with an increased incidence of PHG [19]. There is controversy regarding whether or not PHG that occurs in the context of endoscopic therapy is as clinically relevant as the PHG that occurs "spontaneously". Some studies suggest that PHG that occurs after endoscopic sclerotherapy or ligation therapy is transient [2], whilst another study observed no difference in the natural history of PHG whether or not previous endoscopic sclerotherapy therapy was performed [1]. The natural course of PHG after ligation therapy seems to be milder than after sclerotherapy. The association of beta blockers with endoscopic variceal ligation therapy that is recommended for secondary prophylaxis has been shown to reduce the incidence of PHG [20].

The argon plasma coagulator (APC) is a device that uses argon gas for the delivery of a mono-polar electrical current to a target tissue, used to stop bleeding from ectatic vessels, and to attempt to obliterate the vessels. The combination of a superficial depth of penetration and the non-contact mode of application are ideal for the treatment of mucosal lesions of the gastrointestinal (GI) tract [8].

We applied APC to all areas of visible red spots of congested gastric mucosa with approximately $3-5 \mathrm{~mm}$ distance between APC probe and gastric mucosal lesion for about 1-2 seconds. The end point of successful endoscopic therapy was production of a white coagulum. APC was done in a single session in patients who have localized PHG and in two sessions in patients who have diffuse PHG where endoscopy was repeated after 2 weeks, and further sessions were performed as needed.

On comparing laboratory investigations between 1st visit and one, two and three months in group (A) patients, there was a highly significant increase in hemoglobin level, serum iron and serum ferritin with a significant decrease in TIBC. This means that there was an overall improvement of anemia and iron deficiency. These results may be attributed to coagulation of mucosal vessels thus prevention of bleeding and improvement in gastric perfusion due to decrease in gastric congestion and therefore enhances absorption of the nutrients through gastric mucosa. These results were found to be close to Abd El-Ghany et al. (2014) who reported that, the mean hemoglboin pre-APC of was $8.3 \pm 1.1$ respectively which gradually elevated to become $10.72 \pm 1.54$, this elevation showed significance throughout treatment follow up. Also agree to those reported by Gonzalez-Suarez et al. [18] that studied the effect of APC on bleeding from PHG, where 22 cirrhotic patients (16 patients had PHG with chronic anemia and 6 patients had PHG with acute bleeding episode). APC was applied to the area of PHG. Patients were followed up for a mean of 36 months. Hemoglobin value was significantly improved after APC.

APC has been used successfully to treat vascular bleeding of the upper digestive tract including gastric antral vascular ectasia syndrome [21], sporadic angiodysplasia, hemorrhagic telangiectasia, and radiation-induced enteropathy [22].

A more recent study has challenged the traditional consideration that lesions associated to PHG do not benefit from endoscopic therapy by evaluating the use of argon plasma coagulation (APC) in the treatment of PHG [12]. In this study, 11 patients with bleeding from PHG were included. APC 
sessions were aimed to ablate the greatest area of mucosal surface as possible, at least $80 \%$ in diffuse lesions. Sessions were repeated every 2-4 weeks. The end point, which was defined by the absence of upper $\mathrm{Gl}$ bleeding or a reduction in transfusion requirements, was achieved in $81 \%$ of the patients who were bleeding from PHG.

There were no reported APC complications among the studied groups of patients apart from mild gaseous distension and local pain at epigastrium although these effects are temporary and mild. This agree with Abd El-Ghany et al. who reported that there were no complications related to APC throughout four months' treatment follow up [21].

The primary endpoint of the trial was recurrent hemorrhage from PHG, which was defined by (a) the presence of acute upper gastrointestinal bleeding with a decrease in hematocrit and the finding on emergency endoscopy (performed within the first $12 \mathrm{~h}$ ) of gastric red spots as the source of bleeding, or (b) the presence of chronic bleeding defined by occult blood loss with transfusion requirements of 3 or more units of packed red blood cells in a 3 month period or continuous iron replacement therapy for more than $50 \%$ of the time of follow up. Patients randomized to propranolol remained free of hemorrhage both in the acute hemorrhage ( $85 \%$ vs. $20 \%$ ) and chronic hemorrhage ( $63 \%$ vs. $40 \%$ at 30 months), although the differences were only statistically significant in the former (acute settings) [23]. Multivariate analysis identified the absence of propranolol therapy as the only factor independently predictive of recurrent hemorrhage. The results of these studies led to the consensus recommendation that non-selective beta-blockers should be used in the chronic setting and once the acute episode of bleeding is controlled and the patient is stable [3].

In our study, the use of beta-blockers is associated with the gradual increase in the mean of hemoglobin, serum iron, and serum ferritin and gradual decrease of TIBC these results were found to be close to those reported by Snyder et al. [24].

In our study, there was statistically significant difference between the two groups as regards the changes in hemoglobin and iron after one, two and three months respectively when compared with the first visit that means that the increase in hemoglobin, serum ferritin, serum iron is more in APC group patients than beta-blockers group patients and the decrease in TIBC is more in APC group patients than beta-blockers group patients, so the improvement in hemoglobin and iron study variables is better in APC group patients than detected in betablockers group patients. So these results recommend the use of beta-blockers in controlling the chronic blood loss from congestive gastropathy and this was found close to that was reported by De Franchis [3] and also recommend the use of APC in controlling the chronic blood loss from congestive gastropathy and this was found close to that was reported by Herrera et al., but the improvement in hemoglobin and iron study variables is better in APC group patients than detected in beta-blockers group patients [12].

The "congestion index" is the ratio of portal vein crosssectional area $(\mathrm{cm} 2)$ to mean portal vein flow velocity $(\mathrm{cm} /$ sec), thereby taking into account portal vein dilatation and decreased flow velocity, the two physiological changes associated with portal hypertension [25].

In the present study, the mean congestion index was 0.18 this was found close to that was reported by Zhang et al. who reported that: the congestion index value above 0.1 suggests the diagnosis of portal hypertension with a $95 \%$ sensitivity and specificity and in normal subjects this ratio is approximately 0.07 [26]. Hussain et al. reported that: congestion index of portal vessels is sensitive and specific parameter in the diagnosis of portal hypertension [14].

At the end of the study time we divided patients to two groups according to occult blood in stool test, bleeder group (+ve test) and non-bleeder group (-ve test) we found that there is high significant correlation was found between high congestion index and bleeding. This result was in accordance to Carneiro et al. who conducted a study on 40 patients to assess hemodynamic changes in portal circulation by Doppler sonography after endoscopic sclerotherapy, and concluded that, the higher congestion index the more recurrent attacks of hemorrhage regardless endoscopic sclerotherapy [27].

In our study the mean of congestion index in bleeder group $0.26 \pm 0.06(\mathrm{~cm} \times \mathrm{sec})$ which was highly significant higher than $0.16 \pm 0.02(\mathrm{~cm} \times \mathrm{sec})$ in non-bleeder group this may be close related to Abdel Eslam et al., who found that the congestion index of mean portal vein was significantly higher in bleeders $0.26 \pm 0.08(\mathrm{~cm} \times \mathrm{sec})$ than in patients who have not yet bled $0.18 \pm 0.06(\mathrm{~cm} \times \mathrm{sec})$ [28]. and also, close related to Wahib et al., who reported that congestion index of subgroup suffered from recurrent bleeding was $0.22 \pm 0.02(\mathrm{~cm} \times \mathrm{sec})$ was highly significant higher than those of patients did not suffer from recurrent bleeding $0.12 \pm 0.01(\mathrm{~cm} \times \mathrm{sec})$ [29].

In our study at a cutoff limit of congestion index of portal vein $0.24(\mathrm{~cm} \times \mathrm{sec})$ the sensitivity is $83 \%$ and specificity is $96 \%$ in discriminating bleeder from non-bleeder group. However, when Wahib et al. choose a cutoff limit of $0.16(\mathrm{~cm} \times \mathrm{sec})$ the congestion index of mean portal vein the sensitivity was $100 \%$ and $40.5 \%$ specificity [30]. Although in our study when we choose the same cutoff limit $0.16(\mathrm{~cm} \times \mathrm{sec})$ the sensitivity is $100 \%$ and specificity $64 \%$. However, choose a cutoff limit $(0.13$ $\mathrm{cm} \times \mathrm{s}$ ) in discriminating bleeders group from non-bleeders group, so the sensitivity was (10\%) only, specificity was $13 \%$ [28].

\section{Conclusion}

In short, we can conclude that, the use of either argon plasma coagulation (APC) or beta-blockers in controlling the chronic blood loss from congestive gastropathy shows significant improvement in hemoglobin and iron study variables with both but the significant improvement in APC group patients than in beta-blockers group patients recommended the use of APC to control the chronic blood loss from congestive gastropathy. Multiple sessions of APC are probably needed to achieve complete and sustained endoscopic improvement of severe PHG together with improvement of iron deficiency anemia. APC is safe in 
achieving endoscopic hemostatic purposes. The severity of portal hypertensive gastropathy and congestion index can predict bleeding from gastropathy.

\section{References}

1. Gjeorgjievski M, Cappell MS (2016) Portal hypertensive gastropathy: A systematic review of the pathophysiology, clinical presentation, natural history and therapy. World J Hepatol 8: 231-262.

2. Elwakil R, Al-Breedy AM, Gabal HH (2016) Effect of endoscopic variceal obliteration by band ligation on portal hypertensive gastro-duodenopathy: Endoscopic and pathological study. Hepatol Int 10: 965-973.

3. De Franchis R (2005) Evolving consensus in portal hypertension. Report of the Baveno IV consensus workshop on methodology of diagnosis and therapy in portal hypertension. J Hepatol 43: 167-176.

4. Bang CS, Kim HS, Suk KT, Kim SE, Park JW (2016) Portal hypertensive gastropathy as a prognostic index in patients with liver cirrhosis. BMC Gastroenterol 16: 93.

5. Kumar A, Mishra SR, Sharma P, Sharma BC, Sarin SK (2010) Clinical, laboratory, and hemodynamic parameters in portal hypertensive gastropathy: a study of 254 cirrhotics. J Clin Gastroenterol 44: 294-300.

6. Han S, Chaudhary N, Wassef W (2015) Portal hypertensive gastropathy and gastric antral vascular ectasia. Curr Opin Gastroenterol 31: 506-512.

7. Vignali C, Bargellini I, Grosso M, Passalacqua G, Maglione F (2005) TIPS with expanded polytetrafluoroethylene-covered stent: results of an Italian multicenter study. AJR Am J Roentgenol 185: 472-480.

8. Sato T, Yamazaki K, Toyota J, Karino Y, Ohmura T (2005) Efficacy of argon plasma coagulation for gastric antral vascular ectasia associated with chronic liver disease. Hepatol 32: 121-126.

9. Zakareya T, Omar N, Abu Gabal M (2006) Endoscopic argon plasma coagulation for the treatment of portal hypertensive gastropathy: Short term effects on chronic blood loss. IASL/ AFASLD 2006 Cairo, Egypt. p: 35.

10. Siramolpiwat S (2014) Transjugular intrahepatic portosystemic shunts and portal hypertension-related complications. World J Gastroenterol 20: 16996-17010.

11. Clarke JO, Thuluvath PJ (2007) Endoscopic frontiers in the field of hepatology. Minerva Gastroenterologica e Dietologica 53: 101-109.

12. Herrera S, Bordas JM, Llach J, Ginès A, Pellisé M (2008) The beneficial effects of argon plasma coagulation in the management of different types of gastric vascular ectasia lesions in patients admitted for GI hemorrhage. Gastrointest Endosc 68: 440-446.

13. Pugh RN, Murray-Lyon IM, Dawson JL (1973) Transection of the oesophagus for bleeding oesophageal varices. $\mathrm{Br} J$ Surg 6: 646-649.

14. Hussain $Q$, Badruddin AH, Chaudhry MA, Ahmad F, Abbasi A (2010) Effect of carvedilol on portal pressure estimated by hepatic vein Doppler ultrasound waveform and damping index in cirrhotic patients. J Coll Physicians Surg Pak 20: 586-589.
15. De Franchis $R$ (2000) Updating consensus in portal hypertension: Report of the Baveno III Consensus Workshop on definitions, methodology and therapeutic strategies in portal hypertension. J Hepatol 33: 846-852.

16. El-Zanaty F, Way A (2008) MACRO International. Egypt demographic and Heath Survey, 2008. Final report. In: Measure DHS.

17. Sievert W, Altraif I, Razavi H (2011) A systematic review of HCV epidemiology in Asia, Australia and Egypt. Liver Int 31: 61-80.

18. Fontana RJ, Sanyal AJ, Mehta S, Doherty MC, NeuschwanderTetri BA (2006) Portal hypertensive gastropathy in chronic hepatitis $C$ patients with bridging fibrosis and compensated cirrhosis: results from the HALT-C trial. Am J Gastroenterol 101: 983-992.

19. El-Khayat HR, El-Khattib A, Nosseir M (2010) Portal hypertensive entropathy before and after varriceal oblitration : an endoscopic, histopathologic and immunohistochemical study. J Gastrointestinal Liver Dis 19: 175-179.

20. Garcia-Tsao G, Bosch J (2010) Management of varices and variceal hemorrhage in cirrhosis. N Engl J Med 362: 823-832.

21. Gonzalez B, Villanueva C, Planella M (2003) Endoscopic treatment with argon plasma coagulation for portal hypertensive gastropathy. J Hepatol 38: 59.

22. Abd El-Ghany S, El-taher S, El-Zefzafy W, Sabal A (2014) safety and efficacy of endoscopic treatment with argon plasma coagulation of gastric bleeding in cases of gastric antral vascular ectasia and portal hypertensive gastropathy. AAMJ (12) no (1).

23. Sato Y, Takayama T, Takahari D, Sagawa T, Sato T (2008) Successful treatment for gastro-intestinal bleeding of OslerWeber-Rendu disease by argon plasma coagulation using double-balloon enteroscopy. Endoscopy 2: E228-E229.

24. Chung WJ (2014) Management of portal hypertensive gastropathy and other bleeding. Clin Mol Hepatol 20: 1-5.

25. Snyder P, Ali R, Poles M, Gross SA (2015) Portal hypertensive gastropathy with a focus on management. Expert Rev Gastroenterol Hepatol 9: 1207-1216.

26. Al-Nakshabandi $\mathrm{N}$ (2006) The role of ultrasonography in portal hypertension. Saudi Journal of Gastroenterology 12: 111.

27. Zhang L, Yin J, Duan Y, Yang Y, Yuan L (2011) Assessment of intrahepatic blood flow by Doppler ultrasonography: relationship between the hepatic vein, portal vein, hepatic artery and portal pressure measured intraoperatively in patients with portal hypertension. BMC Gastroenterol 11: 84 .

28. Carneiro FO, Retes FA, Matuguma SE, Albers DV, Chaves DM (2016) Role of EUS evaluation after endoscopic eradication of esophageal varices with band ligation. Gastrointest Endosc 84: 400-407.

29. Eslam M, Ampuero J, Jover M, Abd-Elhalim H, Rincon D (2013) Predicting portal hypertension and variceal bleeding using noninvasive measurements of metabolic variables. Ann Hepatol 12: 588-598.

30. Wahib A, Negm I, El-Dorry A, Abdel-hafez M, Mansour R (2001) Congestion Index "Cl" and Doppler Sonoscores "DSS"of portal vessels as predictors of Recurrent Variceal Bleeding. Sc. J Az. Med. Fac (Girls) 22: 175-180. 